\title{
Statistical Learning for Optimal Control of Hybrid Systems
}

\author{
Jorge Piovesan, Chaouki Abdallah, Magnus Egerstedt, Herbert Tanner and Yorai Wardi
}

\begin{abstract}
In this paper we explore a randomized alternative for the optimization of hybrid systems' performance. The basic approach is to generate samples from the family of possible solutions, and to test them on the plant's model to evaluate their performance. This result is obtained by first presenting the general hybrid optimal control problem, and then converting it into an optimization problem within a statistical learning framework. The results are applied to examples already existing in the literature, in order to highlight certain operational aspects of the proposed methods.
\end{abstract}

\section{INTRODUCTION}

A great effort has been devoted to developing optimal control theory for hybrid systems, extending gradient techniques [4], [5], [7], [10], [21], dynamic programming theory [11], and Pontryagin's Minimum Principle [15], [16]. However, the general hybrid optimal control problem is far from trivial, so various restrictions have been imposed in order to obtain meaningful, and computationally feasible results.

One approach is to limit the control inputs to the discrete domain. Thus, a performance function is optimized by choosing the modal sequence and the corresponding switching times. This idea was proposed in [10], [21] restricting the attention to the switching times using a fixed modal sequence. The result in [10] was later modified [1] to vary the sequence by iteratively inserting new modes and optimizing a new fixed sequence until no further improvements were achieved. The same idea was pursued in [5], with the system restricted to two modes only, and in [4] with the attention concentrated on systems with linear continuous dynamics ( [1], [5], [10], [21] dealt with nonlinear dynamics). Other methods use nonsmooth optimization [14], switching surfaces optimization [7], model predictive control for optimization of continuous inputs [3], and game theoretic approaches [18].

An alternative to classical methods is provided by statistical learning algorithms [17], [19]. The objective of such methods is to optimize a performance measure on average, guaranteeing that the error between the obtained solution and the optimal one is arbitrarily small with probability arbitrarily close to one. This is achieved by sampling the set of potential solutions, and choosing the sample that yields

J. Piovesan (corresponding author) and C. Abdallah are with the Department of Electrical and Computer Engineering of the University of New Mexico, Albuquerque NM 87131, \{jlpiovesan,chaouki\}@ece.unm.edu. M. Egerstedt and Y. Wardi are with the School of Electrical and Computer Engineering at the Georgia Institute of Technology, Atlanta, GA 30332, \{magnus,ywardi\}@ece.gatech.edu. H. Tanner is with the Department of Mechanical Engineering of the University of New Mexico, Albuquerque NM 87131, tanner@unm.edu.

J. Piovesan and C. Abdallah are partially supported by NSF award CNS 0626380 under the FIND initiative. M. Egerstedt and Y. Wardi are supported in part by NSF under Grant \#0509064. H. Tanner is supported by the NSF Career award \#0447898 the best performance. Randomized techniques have been previously used to solve some robust control problems that are NP-hard [12], [20] and for reachability analysis of hybrid systems [6]. The advantage of statistical methods is that they simplify the analysis and design tasks at the cost of not being able to guarantee the optimality of the solution.

We show in this paper that randomized approaches can also be pursued to study optimal control of hybrid systems, as previously discussed in [9] using a simulation example. We present a procedure to use randomized algorithms, obtained in a manner similar to [20], to solve a class of optimization problems for hybrid systems that may include discrete and continuous control inputs, as well as autonomous or controlled transitions between modes. An expression that relates the desired properties of the solution to the number of samples needed to obtain them is provided as a mechanism for controlling the trade-off between the computational complexity (number of samples), and the performance of the solution. The algorithm is tested in simulations, and compared to results obtained via gradient procedures, showing that one advantage that the randomized approach (a model-free technique) has over model-based techniques (e.g. gradient techniques) is that the theoretical analysis is simpler, making it applicable for complex situations, at the price of obtaining a solution that is not guaranteed to be the optimal.

\section{A Hybrid Optimal Control Problem}

The hybrid model provided here allows continuous as well as discrete inputs, autonomous and controlled transitions, and discontinuities in the state trajectory, as given in [8], [15]

Definition 1 (Controlled Hybrid Dynamical System [8])

A Controlled Hybrid Dynamical System (CHDS) is a tuple $\mathbf{H}=\left[Q, \boldsymbol{\Sigma}, \mathbf{G}^{A}, \mathbf{R}^{A}, \mathbf{V}, \mathbf{G}^{C}, \mathbf{R}^{C}\right]$ where:

- $Q$ is the set of discrete states.

- $\boldsymbol{\Sigma}=\left\{\Sigma_{q}\right\}_{q \in Q}$ is the collection of dynamical systems with $\Sigma_{q}=\left(X_{q}, \mathcal{T}, f_{q}, U_{q}\right)$, where $X_{q}$ is the continuous state space, $\mathcal{T} \equiv \mathbb{R}^{+}$is the time set, $f_{q}$ is the continuous dynamics, and $U_{q}$ is the set of continuous controls .

- $\mathbf{V}=\left\{V_{q}^{A}\right\}_{q \in Q} \bigcup\left\{V_{q}^{C}\right\}_{q \in Q}$ is the set of discrete transition labels. $v^{A} \in V_{q}^{A}$ determines the next discrete mode for an autonomous transition, and $v^{C} \in V_{q}^{C}$ determines the next mode for a controlled transition.

- $\mathbf{G}^{A}=\left\{G_{q}^{A}\right\}_{q \in Q}$, where $G_{q}^{A}: V_{q}^{A} \rightarrow X_{q}$, is a guard condition for an autonomous jump for each $q \in Q$.

- $\mathbf{G}^{C}=\left\{G_{q}^{C}\right\}_{q \in Q}$, where $G_{q}^{C}: V_{q}^{C} \rightarrow X_{q}$, is a guard condition for a controlled jump for each $q \in Q$.

- $\mathbf{R}^{A}=\left\{R_{q}^{A}\right\}_{q \in Q}$ where $R_{q}^{A}: G_{q}^{A} \times V_{q}^{A} \rightarrow\left\{X_{p}\right\}_{p \in Q}$ is the autonomous transition map. 
- $\mathbf{R}^{C}=\left\{R_{q}^{C}\right\}_{q \in Q}$, where $R_{q}^{C}: G_{q}^{C} \times V_{q}^{C} \rightarrow\left\{X_{p}\right\}_{p \in Q}$ is the controlled transition map.

Finally, $H=\left(\bigcup_{q \in Q} X_{q}\right) \times Q$ is the hybrid state space of $\mathbf{H}$. Note that $\mathbf{V}$ may include the no transition element $\{i d\}$.

The following notation is used: Labels of different modes are denoted as $v_{q}$, elements of discrete sequences as $v_{[i]}$, and continuous time signals as $v(t) . v^{\prime}$ denotes the state $v$ at the end of an active period (just before a transition), while $v$ denotes its state at the beginning of an active period.

A Hybrid Time Trajectory [13], [15] is a finite or infinite sequence $\tau:=\left\{\tau_{[0]}, \tau_{[1]}, \tau_{[2]}, \ldots\right\}$ such that $\tau_{[i]} \leq \tau_{[i+1]}$ for all $i$, where $\tau_{[i]}$ are the times at which the discrete transition from mode $q_{[i-1]}$ to $q_{[i]}$ takes place. A Hybrid Switching Sequence [13], [15] is a finite or infinite sequence $(\tau, \mathbf{v})=\left\{\left(\tau_{[0]}, v_{[0]}\right),\left(\tau_{[1]}, v_{[1]}\right) \ldots\right\}$ of pairs of switching times $\tau_{[i]}$ and discrete transition events $v_{[i]} \in \mathbf{V}$ (autonomous or controlled), where $\tau$ is a hybrid time trajectory, and $\mathbf{v}$ is called a location schedule.

Definition 2 (Hybrid Input and State Trajectory [15]) A hybrid input $\mathcal{I}:=(\tau, \mathbf{v}, \mathbf{u})$ consists of a hybrid switching sequence $(\tau, \mathbf{v})$ and a piecewise continuous input $\mathbf{u}=\left\{u_{[0]}, u_{[1]} \ldots\right\}$, where $u_{[i]} \in U_{[i]}$ for all $i \in \mathbb{N}$ and all $t \in\left[\tau_{[i]}, \tau_{[i]}^{\prime}\right)$. A hybrid state trajectory $\mathcal{H}=(\tau, \mathbf{q}, \mathbf{x})$ consists of hybrid time trajectory $\tau$, a sequence of discrete states $\mathbf{q}=\left\{q_{[0]}, q_{[1]}, q_{[2]}, \ldots\right\}$ and a sequence of absolutely continuous functions $\mathbf{x}=\left\{x_{[0]}, x_{[1]}, x_{[2]} \ldots\right\}$, where $x_{[i]}(t):\left[\tau_{[i]}, \tau_{[i]}^{\prime}\right) \rightarrow X_{[i]}$ for all $i \in \mathbb{N}$ and all $t \in\left[\tau_{[i]}, \tau_{[i]}^{\prime}\right)$.

Definition 3 (Hybrid Execution [13], [15]) An execution of a CHDS is a collection $\mathcal{E}_{H}=(\tau, \mathbf{v}, \mathbf{u}, \mathbf{q}, \mathbf{x})$, composed by a hybrid input $\mathcal{I}$ and a hybrid state trajectory $\mathcal{H}$ such that $(\tau, \mathbf{v}, \mathbf{u}, \mathbf{q}, \mathbf{x})$ satisfies:

- $h_{0}=\left(q_{[0]}, x_{[0]}(0)\right)$ is an initial condition of $\mathbf{H}$

- Continuous Dynamics: For all $t \in\left[\tau_{[i]}, \tau_{[i]}^{\prime}\right), \dot{x}_{[i]}(t)=$ $f_{q_{[i]}}\left(x_{[i]}(t)\right)$ and $x_{[i]}(t) \in X_{q_{[i]}}$ for all $i \in \mathbb{N}$;

- Discrete Dynamics (Autonomous or Controlled): For all $i \in \mathbb{N}, q_{[i+1]}=v^{*}\left(q_{[i]}\right) \in V_{q_{[i]}}^{*}, x_{[i]}\left(\tau_{[i]}^{\prime}\right) \in G_{q_{[i]}}^{*}$, and $\left(q_{[i+1]}, x_{[i+1]}\left(\tau_{[i+1]}\right)\right) \in R_{q_{[i]}}^{*}$.

Where $(\cdot)^{*}$ denotes $(\cdot)^{A}$ or $(\cdot)^{C}$ depending on the type of dynamics (autonomous or controlled respectively).

We now define the optimal control problem. Let $\left\{l_{[i]}\right\}_{i \in \mathbb{N}}$ be a family of continuous flow cost functions with $l_{[i]}: X_{[i]} \times$ $U_{[i]} \rightarrow \mathbb{R}^{+}$. Let $\left\{c_{a,[i]}\right\}_{i \in \mathbb{N}}$ be a family of autonomous jump costs with $c_{a,[i]}: G_{[i]}^{A} \times V_{[i]}^{A} \rightarrow \mathbb{R}^{+}$. Let $\left\{c_{c,[i]}\right\}_{i \in \mathbb{N}}$ be a family of controlled jump costs with $c_{c,[i]}: G_{[i]}^{C} \times V_{[i]}^{C} \rightarrow \mathbb{R}^{+}$. Let $c_{f}$ be the terminal cost function $c_{f}: Q \times\left\{X_{q}\right\}_{q \in Q} \rightarrow$ $\mathbb{R}^{+}$. Let the hybrid cost function $J\left(t_{0}, t_{f}, h_{0}, \mathcal{I}\right)=J(\mathcal{I})$ be:

$$
J(\mathcal{I}):=\sum_{i=0}^{L}\left(\int_{t_{[i]}}^{t_{[i+1]}} l_{[i]}(s) d s+c_{a,[i]}+c_{c,[i]}\right)+c_{f}
$$

where $\left[t_{0}, t_{f}\right]$ is the optimization interval (assumed finite), $h_{0}$ is the initial condition of the system, and $\mathcal{I}$ is the hybrid input. The optimal control problem can then be stated as:
$\mathcal{P} 1$ [15]: Given a system $\mathbf{H}$ (Definition 1) with hybrid execution $\mathcal{E}_{H}$ (Definition 3), optimization interval $\left[t_{0}, t_{f}\right]$, and initial condition $h_{0}$, the Hybrid Optimal Control Problem (HOCP) is to minimize the total cost (1), over the family of input trajectories $\{\mathcal{I}\}$. If a hybrid input $\mathcal{I}^{*}$ minimizes $J(\mathcal{I})$, then it is called a hybrid optimal control for $\mathbf{H}$.

\section{STATistical Learning Theory}

Suppose you have a system with decision vector $f \in \mathcal{F}$ and cost functional $R: \mathcal{F} \rightarrow \mathbb{R}$, and consider the problem of estimating the best performance of the system $R^{*}=$ $\inf _{f \in \mathcal{F}} R(f)$. For this purpose $N$ independent and identically distributed (i.i.d.) random samples $f_{i} ; i=1, \ldots, N$ are taken from $\mathcal{F}$ according to a probability distribution $P(f)$. Then the sample minimum is defined as $R^{0}=\min _{i=1, \ldots, N} R\left(f_{i}\right)$, and the objective becomes that of making $R^{0}$ as close as possible to $R^{*}$ [17]. This leads to:

Definition 4 (Probable Near Minimum) Given $R(f), \delta \in$ $(0,1), \alpha \in(0,1)$, a number $R_{0} \in \mathbb{R}$ is said to be a probable near minimum of $R(f)$ to level $\alpha$ and confidence $1-\delta$ if there exists a set $\tilde{\mathcal{F}} \subseteq \mathcal{F}$ with $\operatorname{Pr}\{\tilde{\mathcal{F}}\} \leq \alpha$ such that

$$
\operatorname{Pr}\left\{\inf _{\mathcal{F}} R(f) \leq R_{0} \leq \inf _{\mathcal{F} \backslash \tilde{\mathcal{F}}} R(f)\right\} \geq 1-\delta
$$

Loosely speaking, the level $\alpha$ describes a set of potential solutions that may not be represented in the samples taken for optimization. If this set is made small there will be a small probability of finding a better solution than the sample minimum. The confidence $(1-\delta)$ describes the probability of obtaining the desired level. Definition 4 then implies that if $\alpha$ and $\delta$ are made small (but different than zero), the probability of finding a better solution will be small $(\alpha)$, with high confidence $(1-\delta)$.

The mechanism to control the level and confidence of the sample minimum is the number of samples $N$ taken from the set of possible solutions. In order to obtain a probable near minimum (Definition 4) from the samples, $N$ can be obtained as a function of $\alpha$ and $\delta$ using the following Lemma [19]:

Lemma 1 (Lemma 11.1 [19]) Suppose $(\mathcal{F}, \mathcal{S}, P)$ is a probability space ${ }^{1}$, and that $R: \mathcal{F} \rightarrow \mathbb{R}$ is a random variable. Let $f_{1}, \ldots, f_{N} \in \mathcal{F}$ be $N$ i.i.d. samples drawn according to $P$, and define

$$
R^{0}(\mathbf{f})=\min _{1 \leq i \leq N} \nu\left(f_{i}\right)
$$

and $p_{f}=P\left\{f \in \mathcal{F}: R(x)<R^{0}(\mathbf{f})\right\}$, then if $0<\alpha<1$

$$
P^{N}\left\{\mathbf{f} \in \mathcal{F}^{m}: p_{f}>\alpha\right\} \leq(1-\alpha)^{N}
$$

In words, this result estates that for a sufficiently large number of samples $N$, the probability that the sample minimum (3) is close to the optimal solution, will be close to one. Detailed coverage of theory on Randomized Algorithms or Statistical Learning can be found in [17], [19].

\footnotetext{
${ }^{1} \mathrm{P}$ differs from $\mathrm{Pr}$ in that the former is assumed to satisfy the properties of a probability measure
} 


\section{RANDOMIZED OptimizATION OF HybRID SYSTEMS}

In order to use learning theory to solve optimization problems for hybrid systems, we restate the HOCP $(\mathcal{P} 1)$ as a statistical learning one. So instead of looking for a solution $\mathcal{I}^{*}$ that guarantees that the cost function (1) achieves its absolute minimum, we seek an approximate $\mathcal{I}^{0}$ that evaluates (1) arbitrarily close to its minimum with probability almost equal to one. The Randomized HOCP can be stated as:

$\mathcal{P} 2$ : Given a level parameter $\alpha \in(0,1)$ and a confidence parameter $\delta \in(0,1)$. The Randomized HOCP is to find a hybrid input $\mathcal{I}^{0}$ such that $J\left(\mathcal{I}^{0}\right)$ is a probable near minimum (Definition 4) with level $\alpha$ and confidence $1-\delta$ of the actual minimum $J\left(\mathcal{I}^{*}\right)(\mathcal{P} 1)$.

\section{Assumption 1 We assume that:}

- There is no uncertainty in the dynamics of the system.

- The plant only allows for controlled transitions, i.e. in Definition $1 \mathbf{V}=\left\{V_{q}^{C}\right\}_{q \in Q}, \mathbf{G}^{A}=\{\}$, and $\mathbf{R}^{A}=\{\}$ for all $q \in Q$; and the Executions $\mathcal{E}_{H}$ (Definition 3) do not contain autonomous discrete dynamics.

We initially restrict our attention to plants without uncertainties. This simplifies the problem at the learning level because this assumption states the the system's model is perfectly known, implying that the estimation of the probability distribution of the plant (done in [12], [20]) is not needed. We also restrict ourselves to plants that allow only controlled transitions. However most of the work in modelbased optimization makes the same assumption [1], [4], [5], [14], [21]. Note also that we consider systems with both continuous and discrete inputs.

Denote by $\{\hat{\mathcal{I}}\}$ the set of the input samples $\left\{\hat{\mathcal{I}}_{1}, \ldots \hat{\mathcal{I}}_{N}\right\}$ that will be used to estimate the optimal hybrid input. Let

$$
J^{*}=\inf _{\mathcal{I} \in\{\mathcal{I}\}} J(\mathcal{I})=J\left(\mathcal{I}^{*}\right)
$$

be the minimum cost value for the system over the complete family of hybrid inputs $\{\mathcal{I}\}$ (true optimum), and let

$$
J^{0}=\min _{1 \leq i \leq N} J\left(\hat{\mathcal{I}}_{i}\right)=J\left(\mathcal{I}^{0}\right)
$$

be the minimum cost value for the system over the set of input samples $\{\hat{\mathcal{I}}\}$. We then have the following results.

Theorem 1 (Minimum number of input samples) The minimum number of samples $N$ that guarantee that $J^{0}$ is a probable near minimum to level $\alpha$ and confidence $\delta$ of $J^{*}$ is

$$
N \geq \frac{\ln (1 / \delta)}{\ln (1 /(1-\alpha))}
$$

Proof: ${ }^{2}$ In Definition 4 let $R_{0}$ be $J^{0}$ and $R(f)$ be $J(\mathcal{I})$. Then (2) can be expressed as

$$
\operatorname{Pr}\left\{J^{0} \in\left[\inf _{\{\mathcal{I}\}} J(\mathcal{I}), \inf _{\{\mathcal{I}\} \backslash\{\tilde{\mathcal{I}}\}} J(\mathcal{I})\right]\right\} \geq 1-\delta
$$

with $\operatorname{Pr}\{\{\tilde{\mathcal{I}}\}\} \leq \alpha$, where $\{\tilde{\mathcal{I}}\} \subseteq\{\mathcal{I}\}$.

\footnotetext{
${ }^{2}$ Proof adapted from Tempo et al. [17] Theorem 9.1 for our problem
}

This expression is equivalent to

$$
\operatorname{Pr}\left\{J^{0} \notin\left[\inf _{\{\mathcal{I}\}} J(\mathcal{I}), \inf _{\{\mathcal{I}\} \backslash\{\tilde{\mathcal{I}}\}} J(\mathcal{I})\right]\right\} \leq \delta
$$

with $\operatorname{Pr}\{\{\tilde{\mathcal{I}}\}\} \leq \alpha$.

Since $\operatorname{Pr}\left\{J^{0}<\inf _{\{\mathcal{I}\}} J(\mathcal{I})\right\}=0$ the inner part of (8) $J^{0} \notin J^{0}\left[\inf _{\{\mathcal{I}\}} J(\mathcal{I}), \inf _{\{\mathcal{I}\} \backslash\{\tilde{\mathcal{I}}\}} J(\mathcal{I})\right]$ with $\operatorname{Pr}\{\{\tilde{\mathcal{I}}\}\} \leq \alpha$ is equivalent to $\operatorname{Pr}\left\{\mathcal{I} \in\{\mathcal{I}\}: J(\mathcal{I})<J^{0}\right\}>\alpha$, where $J^{0}$ is obtained from (6). Let $\mathbf{I}=\left(\hat{\mathcal{I}}_{1}, \hat{\mathcal{I}}_{2}, \ldots \hat{\mathcal{I}_{N}}\right)$, so (8) can be expressed (due to the i.i.d assumption on $\hat{\mathcal{I}_{1}}, \hat{\hat{I}_{2}}, \ldots . \hat{\mathcal{I}_{N}}$ ) as

$$
\operatorname{Pr}^{N}\left\{\mathbf{I} \in\{\hat{\mathcal{I}}\}^{N}: \operatorname{Pr}\left\{\mathcal{I} \in\{\mathcal{I}\}: J(\mathcal{I})<J^{0}\right\}>\alpha\right\}
$$

Comparing (9) to (4) we note that it is upper-bounded by $(1-\alpha)^{N}$. Since we want (9) to be smaller than $\delta,(8)$ implies $(1-\alpha)^{N} \leq \delta$, which implies (7).

Algorithm 1 (Randomized optimization of hybrid input) Given a desired level $\alpha$ a desired confidence $1-\delta$ and the complete description of the system (Definition 1):

1) Calculate the minimum number of samples $N$ needed to obtain a probable near minimum (p.n.m.) using (7)

2) Generate $N$ i.i.d. input samples $\hat{\mathcal{I}_{1}}, \hat{\mathcal{I}_{2}}, \ldots \hat{\mathcal{I}_{N}}$ from the family of all possible hybrid inputs $\{\mathcal{I}\}$

3) Test each input sample $\hat{\mathcal{I}}_{i}$ for $i=1,2, \ldots, N$ in the plant and calculate their performance $J\left(\hat{\mathcal{I}}_{i}\right)$.

4) The p.n.m. cost $J^{0}$ is given by (3) and the p.n.m. hybrid input is $\mathcal{I}^{0}=\arg \min _{\hat{\mathcal{I}_{1}}, \hat{\mathcal{I}}_{2}, \ldots \hat{\mathcal{I}_{N}}} J\left(\hat{\mathcal{I}}_{i}\right)$.

Remark 1 (On the hybrid switching sequence) The procedure to sample hybrid switching sequences $(\tau, \mathbf{v})$ can vary according to each particular problem. If a switching sequence has to be obtained directly, a promising procedure is composed of two steps: First, randomly select a desired number of transitions $\vartheta$ for the sample, and then pick a random switching sequence $(\tau, \mathbf{v})$ of $\vartheta+1$ elements, with the times inside the optimization interval $\left[t_{0}, t_{f}\right]$, and the transition events chosen from V. Note that one can pick any distribution for number of transitions or the switching sequence, while keeping the number of transitions bounded.

Remark 2 (On the piecewise continuous input) Since on each mode interval the continuous part of the hybrid input must be continuous, one could generate a large number of classes of input signals using a set of parameterized basis functions (e.g. linear, sinusoidal, exponential), such that during the sampling, their parameters and their weights are chosen to obtain the final input signal. Another option may be to express the continuous input as a parameterized (feedback) function of the state.

\section{A. An application example}

We provide an example to test and clarify the proposed approach. For comparison purposes, we use an example that already exists in the literature. In this example we optimize the performance of a dynamical system by choosing the hybrid switching sequence. There is no continuous component in the hybrid input of the existing example [2]. 


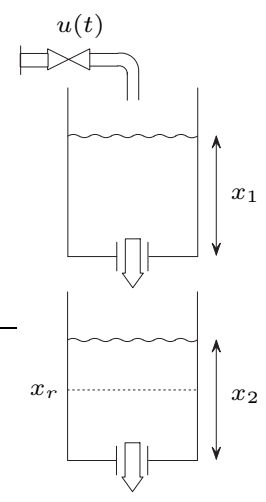

Fig. 1. Graphical description of the tank system.

The system is composed of two tanks, where the objective is to control the fluid level of the second tank via the input flow rate to the first tank (Figure 1). The input variable $u(t)$ is the input flow rate to the first tank, and the state variables $x_{1}(t)$ and $x_{2}(t)$ are the fluid levels at the first and second tanks respectively. Defining $x(t)=\left[x_{1}(t), x_{2}(t)\right]^{T}$ the system's equations (by Torricelli's principle) are:

$$
\dot{x}=f(x, u)=\left[\begin{array}{c}
-\gamma_{1} \sqrt{x_{1}}+u \\
\gamma_{1} \sqrt{x_{1}}-\gamma_{2} \sqrt{x_{2}}
\end{array}\right]
$$

where $\gamma_{1}, \gamma_{2}>0$ are fixed constants. The control input is constrained to three operating states: fully open, half open, and fully closed, i.e, $u(t) \in\left\{u_{\max }, \frac{1}{2} u_{\max }, 0\right\}$, for some $u_{\max }>0$. Since these are discrete values, each operating state generates a discrete mode $q \in Q$ in a hybrid model (Definition 1). If the set of locations is $Q=$ $\{1,2,3\}$, the dynamical systems $\Sigma_{1}, \Sigma_{2}, \Sigma_{3}$ are described as: $f_{1}(x, u)=f\left(x, u_{\max }\right), f_{2}(x, u)=f\left(x, \frac{1}{2} u_{\max }\right)$, and $f_{3}(x, u)=f(x, 0), X_{1}=X_{2}=X_{3}=\left\{x \in \mathbb{R}^{2}: x_{1} \geq\right.$ $\left.0, x_{2} \geq 0\right\}$, and $U_{1}=U_{2}=U_{3}=\{\} \cdot V_{q}^{C}=Q$ for all $q \in Q$ (no restrictions on the mode transitions), $G_{q}^{C}=X_{q}$ for all $q \in Q$ (a discrete transition is possible for any value of $x$ ), and $R_{q}^{C}$ leaves the continuous state unchanged for all $q \in Q$. Note that the system satisfies Assumption 1.

Given an initial condition $x_{0}=x(0)$ and a final time $T>$ 0 , the objective is to select a switching sequence that drives the error between $x_{2}(t)$ and a reference signal $x_{r}(t) \in \mathbb{R}$ to zero. To this end, the cost function is defined as

$$
J=K \int_{0}^{T}\left(x_{2}(t)-x_{r}(t)\right)^{2} d t
$$

for some $K>0$. Comparing (1) to (11), the final cost and the transition costs are zero, and the flow cost is a continuous function (because the state flows are continuous).

The chosen parameters for the simulation (identical to [2]) are $x_{0}=[0.4,0.4]^{T}, T=5, K=10, \gamma_{1}=\gamma_{2}=1$, $u_{\max }=1$, and $x_{r}(t)=0.5+0.25 \frac{t}{T}$. The level and confidence parameters are $\alpha=\delta=0.002$ yielding $N=3105$ samples, generated according to Remark 1 with the number of transitions distributed according to a uniform distribution $U[1,10]$, the transition times according to $U[0, T]$ and the modes according to $U(1,2,3)$. The approximate optimal costs values in three different simulations were 0.1072 , 0.1090 and 0.1058 . The switching sequence and the state trajectories for $J^{0}=0.1058$ are shown in Figures 2 and 3 . Comparing this result to that reported in [2], the performance obtained using the proposed approach is very close to that obtained using gradient techniques, where the reported final costs, under the same conditions, were 0.107 and 0.105 [2]. The continuous state trajectories also look similar, even though the mode schedules are different.

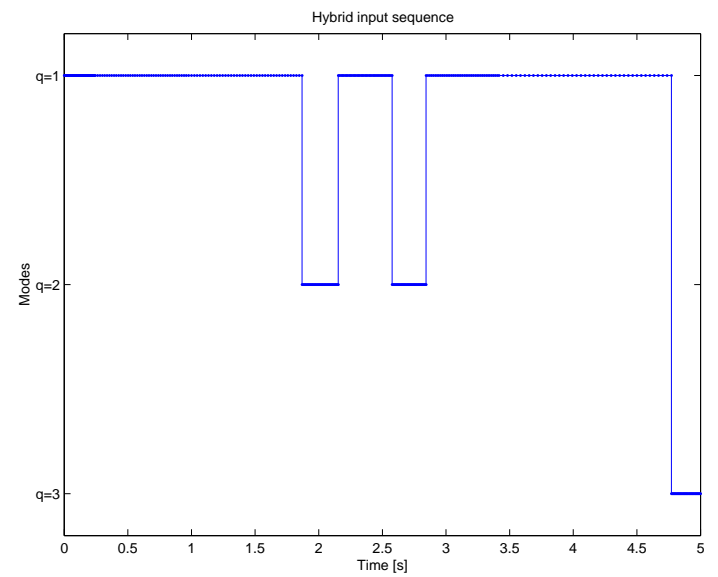

Fig. 2. Optimal mode sequence for tanks problem using the proposed randomized approach.

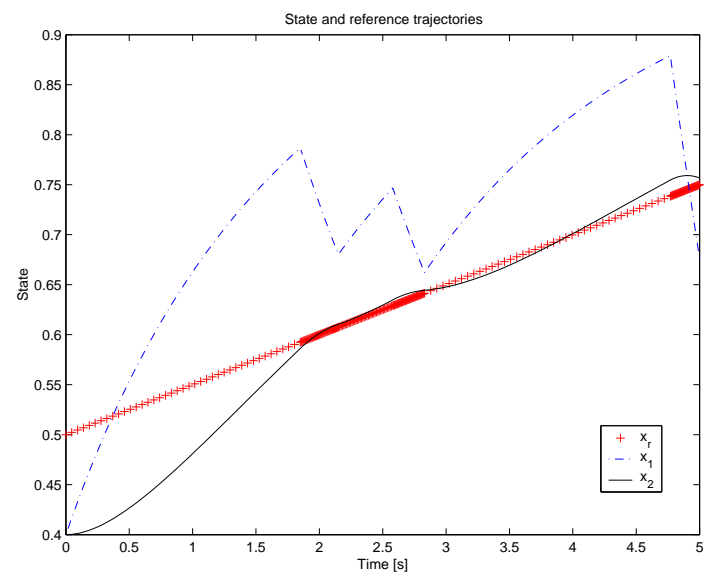

Fig. 3. Optimal state trajectories and reference signal for tanks problem using the proposed randomized approach $\left(x_{r}(t)\right.$ : Reference, $x_{1}(t)$ and $x_{2}(t)$ : State trajectories).

\section{RELAXING ASSUMPTION 1}

In this section we show how the procedure explained above can be applied to systems that instead of having controlled transitions, are subject to autonomous transitions triggered by internal events. We also discuss how we can extend this procedure for the control uncertain systems.

\section{A. Systems with internal autonomous transitions}

In order to study systems with purely internal autonomous transitions we modify Assumption 1 as follows: 
Assumption 2 We assume that:

- There is no uncertainty in the dynamics of the system.

- The plant only allows for autonomous transitions. i.e. in Definition $1 \mathbf{V}=\left\{V_{q}^{A}\right\}_{q \in Q}, \mathbf{G}^{C}=\{\}$, and $\mathbf{R}^{C}=\{\}$ for all $q \in Q$; and the Executions $\mathcal{E}_{H}$ (Definition 3) do not contain controlled discrete dynamics. Furthermore, the autonomous transitions are completely determined by the dynamics of the system.

The second point in Assumption 2 implies that when a guard condition in $\mathbf{G}^{A}$ is satisfied during the system's operation, that transition must occur immediately (forced transition). Note that the hybrid input of the system can not be controlled directly. However a special type of hybrid input is generated and completely determined by the autonomous discrete dynamics. Thus if these dynamics were modified, different hybrid inputs could be generated.

The procedure to modify these dynamics is to consider the transition guards and reset maps in $\mathbf{G}^{A}$, and $\mathbf{R}^{A}$ as control variables. Thus Theorem 1 and Algorithm 1 may be applied to autonomous discrete dynamics if instead of sampling hybrid inputs directly, one generates samples for the switching guards, reset maps, and feedback functions of continuous state of the system, generating hybrid input samples indirectly.

\section{B. An example of autonomous transitions optimization}

This example was previously presented in [7]. The problem consists of a unicycle mobile robot, that has to reach a point in the plane departing from an initial condition while avoiding a point obstacle. The robot may be controlled by switching between two discrete behaviors, "approach goal" (mode 1) and "avoid obstacle" (mode 2). The obstacle is surrounded by two circular guards (centered at the obstacle position) that determine which modes are active. If the robot is in mode 1 and reaches the inner guard, it switches to mode 2 . If it is in mode 2 and reaches the outer guard, it switches to mode 1 . The optimization problem is to choose the best radii for these two circular guards such that the robot gets as close as possible to the goal without hitting the obstacle.

The robot is described using its kinematic model as:

$$
\begin{aligned}
\dot{x} & =v \cos \phi \\
\dot{y} & =v \sin \phi \\
\dot{\phi} & =\omega
\end{aligned}
$$

where the position of the robot $(x, y)$ and its orientation $\phi$ form the continuous state of the system, and $v$ and $\omega$ are its linear and angular speeds. The goal is located at $\left(x_{g}, y_{g}\right) \in$ $\mathbb{R}^{2}$, and the obstacle at $\left(x_{o}, y_{o}\right) \in \mathbb{R}^{2} . v$ has a constant value while $\omega$ is the feedback control input that changes according to each mode: In mode $1, \omega$ is given by $\omega_{1}=C_{1}\left(\phi_{g}-\phi\right)$ where $\phi_{g}=\arctan \left(\left(y_{g}-y\right) /\left(x_{g}-x\right)\right)$ and $C_{1}>0$ is a constant. In mode $2, \omega$ is given by $\omega_{2}=C_{2}\left(\phi-\phi_{o}\right)$ where $\phi_{o}=\arctan \left(\left(y_{o}-y\right) /\left(x_{o}-x\right)\right)$ and $C_{2}>0$ is a constant.

The procedure to map the robot model (12) to the hybrid system (Definition 1) is similar to that on the previous example. However the parametrization of the switching guards and reset maps is different (similarly to [7] we assume a fixed continuous control input $\left(C_{1}, C_{2}\right)$ that is not considered as an optimization variable). Note from the nature of the system that the reset maps $R_{q}^{C}$ leave the continuous state unchanged for all $q \in Q$, while the transition guards must be parameterized according to the circular guards described in the problem. Therefore, let

$$
\begin{aligned}
G_{1}^{A}\left(v_{1}=2, r_{\text {in }}\right) & =\left(x_{o}-x\right)^{2}+\left(y_{o}-y\right)^{2}-r_{\text {in }}^{2} \\
G_{2}^{A}\left(v_{2}=1, r_{\text {out }}\right) & =\left(x_{o}-x\right)^{2}+\left(y_{o}-y\right)^{2}-r_{\text {out }}^{2}
\end{aligned}
$$

where the $G_{1}^{A}$ is the guard to jump from mode 1 to mode 2, $G_{2}^{A}$ is to jump from mode 2 to mode 1 , and $r_{\text {in }}$ and $r_{\text {out }}$ are the radii of the inner and outer guards respectively. The cost functional is defined as

$J=\int_{0}^{T}\left[\left(x_{g}-x\right)^{2}+\left(y_{g}-y\right)^{2}+\beta \mathrm{e}^{-\xi\left[\left(x_{o}-x\right)^{2}+\left(y_{o}-y\right)^{2}\right]}\right] d t$.

The simulations parameters are $v=1, C_{1}=2, C_{2}=$ $0.4, T=3, \xi=10, \beta=5,\left(x_{g}, y_{g}\right)=(2.25,2)$, $\left(x_{o}, y_{o}\right)=(1,1)$, and $(x(0), y(0), \phi(0))=(0,0,0)$. The level and confidence parameters are $\alpha=0.02$ and $\delta=0.02$ yielding $N=194$ (note that because the sampling space is simple, low values of level and confidence parameters can yield to good results). The resulting approximate optimal cost was $J^{0}=10.4695$ with optimal $r_{i n}^{0}=0.4807$, $r_{\text {out }}^{0}=0.5006$, while an optimization performed using the algorithm reported in [7] with the same values yielded $J^{*}=10.4609, r_{i n}^{*}=0.4963, r_{\text {out }}^{*}=0.4963$. A comparison of the trajectories and guards obtained via the randomized approach (Figure 4), and those obtained using the gradient descent approach [7] (Figure 5) shows that both results are almost identical.

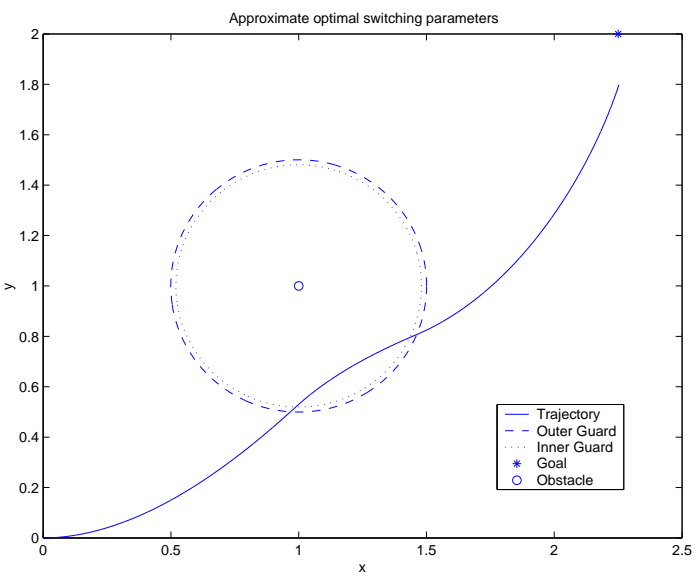

Fig. 4. Robot trajectory and optimal guards using the proposed randomized approach

\section{Possible extensions for uncertain systems}

Statistical learning is useful in the control of uncertain systems [12], [17], [20]. Particularly, in the case of hybrid systems, these uncertainties may be encountered in the form of parameter uncertainties, noise, or externally triggered 


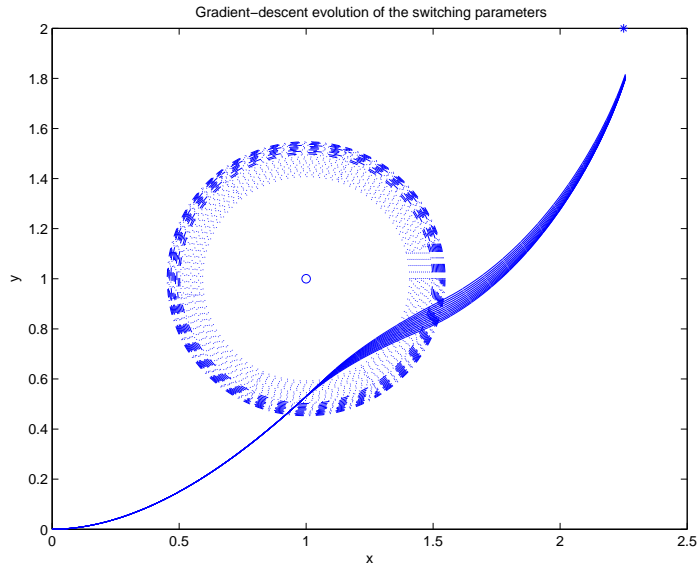

Fig. 5. Evolution of the robot trajectory and switching guards using a gradient descent approach. The optimum configuration is where the two guards collide.

transitions (discrete jumps caused by external events that are not known a priori). Thus consider a hybrid system with any of the two transition types previously treated (controlled or autonomous internally triggered) together with modeling uncertainty. In order to control such system, the approach would be to split the hybrid input $\mathcal{I}$ in two separate sequences, one that can be completely controlled $\mathcal{I}^{c}$ and another representing the uncertainties $\mathcal{I}^{u}$. With these two sequences the goal would be to obtain the average behavior of the uncertain sequence $\mathcal{I}^{u}$ in order to apply the result obtained in this paper (or a similar one) to optimize the expected value of the performance of the system $E\left[J\left(\mathcal{I}^{c}, \mathcal{I}^{u}\right)\right]$ by choosing the best control sequence $\mathcal{I}^{c}$. In this manner, we would guarantee that the system performs optimally on average, with certain accuracy (given for the average part of the procedure), level, and confidence. To this end, we must apply concepts such as Hoeffding inequality [17], or VCtheory [17], [19], together with the ideas explained in this paper.

\section{CONCLUSIONS}

We have presented a randomized approach for optimal control of hybrid systems. We defined a general hybrid optimal control problem, and stated an equivalent problem in the randomized framework. We provided an expression that relates the requirements of the desired solution (level $\alpha$ and confidence $1-\delta$ ) with the computational complexity (number samples) needed to guarantee such requirements. In this form the performance/ computational complexity trade-off can be controlled. We tested our approach on two different examples and obtained comparable results to those available in the literature using model-based approaches. The advantage of the proposed approach is that the theoretical analysis is simplified due to its model-free nature, making it attractive for complicated systems where model-based techniques may face difficulties. However, the price to pay for this problem simplification is that the proposed approach does not guarantee the optimality of the final solution.
The results obtained so far are useful for special subclasses of the general hybrid system defined in the paper, but future research directions include the generalization of the proposed algorithm to be applicable for both types of discrete dynamics, and for control of uncertain systems.

\section{REFERENCES}

[1] H. Axelsson, Y. Wardi, M. Egerstedt, and E. Verriest. A provably convergent algorithm for transition-time optimization in switched systems. In Proceedings of the IEEE Conference on Decision and Control, pages 1387-1402, Seville, Spain, Dec. 2005.

[2] H. Axelsson, Y. Wardi, M. Egerstedt, and E. Verriest. A gradient descent approach to optimal mode scheduling in hybrid dynamical systems. Journal of Optimization Theory and Applications, 2006. Submitted.

[3] A. Bemporad, F. Borrelli, and M. Morari. Piecewise linear optimal controllers for hybrid systems. In Proceedings of the American Control Conference, pages 1190-1195, Chicago, IL, USA, June 2000.

[4] A. Bemporad, A. Giua, and C. Seatzu. Synthesis of state-feedback optimal controllers for continuous-time switched linear systems. In Proceedings of the IEEE Conference on Decision and Control, pages 3182-3187, Las Vegas, NV, USA, Dec. 2002.

[5] S. Bengea and R. DeCarlo. Optimal control of switching systems. Automatica, Elsevier Science Ltd., 41(1):11-27, Jan. 2005.

[6] A. Bhatia and E. Frazzoli. Incremental search methods for reachability analysis of continuous and hybrid systems. In R. Alur and G. Pappas, editors, Proc. of 7th Inter. Workshop, Hybrid Systems: Computation and Control, LNCS 2993, pages 142-156, Berlin, Germany, 2004. Springer-Verlag.

[7] M. Boccadoro, Y. Wardi, M. Egerstedt, and E. Verriest. Optimal control of switching surfaces in hybrid dynamical systems. Journal of Discrete Event Dynamic Systems, 15(4):433-448, Dec. 2005.

[8] M. Branicky, V. Borkar, and S. Mitter. A unified framework for hybrid control: Model and optimal control. IEEE Transactions on Automatic Control, 43(1):31-45, Jan. 1998.

[9] C. Collaro, C. Abdalah, A. Tornambè, and U. Dole. Optimal control of hybrid systems using statistical learning. In Proceedings of the IEEE Conference on Decision and Control, pages 1415-1420, Seville, Spain, December 2005.

[10] M. Egerstedt, Y. Wardi, and H. Axelsson. Transition-time optimization for switched-mode dynamical systems. IEEE Transactions on Automatic Control, 51(1):110-115, Jan. 2006.

[11] S. Hedlund and A. Rantzer. Convex dynamic programming for hybrid systems. IEEE Transactions on Automatic Control, 47(9):1536-1540, Sept. 2002.

[12] V. Koltchinskii, C. Abdallah, M. Ariola, P. Dorato, and D. Panchenko. Improved sample complexity estimates for statistical learning control of uncertain systems. IEEE Transactions on Automatic Control, 45(12):2383-2388, December 2000.

[13] J. Lygeros. Lecture notes on hybrid systems. Notes for an ENSIETA workshop, February-June 2004.

[14] D. Pepyne and C. Cassandras. Optimal control of hybrid systems in manufacturing. Proceedings of the IEEE, 88(7):1108-1123, 2000.

[15] M. Shaikh. Optimal Control of Hybrid Systems: Theory and Algorithms. PhD thesis, Department of Electrical and Computer Engineering, McGill University, Montréal, Canada, 2004.

[16] H. Sussmann. A maximum principle for hybrid optimal control problems. In Proceedings of the IEEE Conference on Decision and Control, pages 425-430, Phoenix, AZ, USA, Dec. 1999.

[17] R. Tempo, G. Calafiore, and F. Dabbene. Randomized Algorithms for Analysis and Control of Uncertain Systems. Communications and Control Engineering. Springer, London, UK, 2003.

[18] C. Tomlin, J. Lygeros, and S. Sastry. A game theoretic approach to controller design for hybrid systems. Proceedings of the IEEE, 88(7):949-970, 2000.

[19] M. Vidyasagar. A Theory of Learning and Generalization. Communications and Control Engineering. Springer, London, UK, 1997.

[20] M. Vidyasagar. Randomized algorithms for robust controller synthesis using statistical learning theory. Automatica, Elsevier Science Ltd., 37:1515-1528, 2001.

[21] X. Xu and P. Antsaklis. Optimal control of switched systems based on parameterization of the switching instants. IEEE Transactions on Automatic Control, 49(1):2-16, Jan. 2004. 